\title{
Burnout as a Mediator in the Relationship between Work-Life Balance and Empathy in Healthcare Professionals
}

\author{
Hwo-Yeon Seo', Dong-Wook Lee ${ }^{2}$, Soohyun $\mathrm{Nam}^{3}$, Sung-jun $\mathrm{Cho}^{4}$, \\ Je-Yeon Yoon ${ }^{5}$, Yun-Chul Hong ${ }^{2}$, and Nami Lee ${ }^{3,6} \bowtie$ \\ ${ }^{1}$ Public Health Medical Service, Seoul National University Hospital, Seoul, Republic of Korea \\ ${ }^{2}$ Department of Preventive Medicine, College of Medicine, Seoul National University, Seoul, Republic of Korea \\ ${ }^{3}$ Human Rights Center, Seoul National University Hospital, Seoul, Republic of Korea \\ ${ }^{4}$ Department of Psychiatry, Kangbuk Samsung Hospital, Seoul, Republic of Korea \\ ${ }^{5}$ Yeongeon Student Support Center, Seoul National University College of Medicine, Seoul, Republic of Korea \\ ${ }^{6}$ Department of Psychiatry, Seoul National University Hospital, Seoul, Republic of Korea
}

Objective This cross-sectional study aimed to 1) explore the relationships among work-life balance (WLB), burnout, and empathy and 2) investigate the roles of the subtypes of burnout relating to WLB and empathy.

Methods A total of 105 health care professionals from a general hospital in Seoul were assessed using the Maslach Burnout Inventory, Jefferson Scale of Physician Empathy, and a one-sentence-question on subjective WLB. Multiple questions on psychiatric problems, including sleep problems, anxiety, depressive symptom, and alcohol problems, were also included.

Results In the mediation analyses, personal achievement was considered as a potential mediating variable between WLB and empathy. The direct effect ( $\beta=3.93,95 \%$ CI: $1.21-6.64)$ and the indirect effect ( $\beta=1.95,95 \%$ CI: $0.52-3.76)$ of WLB on empathy were also significant.

Conclusion Interventions encouraging personal achievement may help mitigate burnout of health professionals.

Psychiatry Investig 2020;17(9):951-959

Key Words Work-life balance, Burnout, Empathy, Healthcare professionals.

\section{INTRODUCTION}

Although Korea has been rapidly industrialized through workers' dedication, subjective well-being that incorporates a balanced life has been largely ignored. Mandatory workloads without quality time with family and friends may cause various mental illnesses, including suicide and other violent tendencies. A balance between work and personal life is essential since the two realms can function as a buffering and compensatory safety space for each other.

Korean workers worked an average of 2,071 hours, 17\% more than the OECD average, while Germans worked 1,301

Received: April 28, 2020 Revised: June 20, 2020

Accepted: July 29, 2020

$\triangle$ Correspondence: Nami Lee, $\mathrm{PhD}$

Department of Psychiatry, Seoul National University Hospital, 103 Daehak-ro, Jongno-gu, Seoul 03080, Republic of Korea

Tel: +82-2-2072-3761, Fax: +82-2-747-4830, E-mail: nami6107@naver.com

(c) This is an Open Access article distributed under the terms of the Creative Commons Attribution Non-Commercial License (https://creativecommons.org/licenses/bync/4.0) which permits unrestricted non-commercial use, distribution, and reproduction in any medium, provided the original work is properly cited. hours in 2015. ${ }^{1}$ In 2018, the work-life balance (WLB) of 30,649 respondents in Korea was analyzed, using the World Health Organization index for well-being. Poor WLB, which is based on the concept of a good fit between working hours and social commitments, was associated with poor psychosocial well-being with low-level job autonomy and being female being the most critical risk factors. ${ }^{2}$ Excessive working hours can also negatively affect sleep duration, impairing productivity, and causing workplace accidents and errors. ${ }^{3}$ An intensive working atmosphere has also been associated with alcohol abuse. ${ }^{4}$ In Korea, a high prevalence of alcohol-related problems has been reported, including neuropsychiatric illness and loss of productivity. ${ }^{5}$ The South Korean Ministry of Health and Welfare estimated alcohol-related social costs to be more than $\$ 21$ billion annually. ${ }^{6}$

Many medical professionals in Korea are burdened by excessive workloads and poor work-life balance. In 2019, the Korean government tried to implement a law for shorter working hours to maintain a better WLB. In 2015, a law that limits residents working in hospitals to 80 working hours was 
enacted. However, the overwhelming number of patients visiting training hospitals still challenges medical professionals' well-being. ${ }^{7}$ In a survey on Korean doctors' satisfaction with their jobs, $51 \%$ of 1,004 physicians were content with their job, but approximately $80 \%$ reported experiencing burnout with physical and mental exhaustion because of excessive workloads. ${ }^{8}$ Studies at Korean hospitals have found higher levels of burnout than among nurses in western countries, with similar effects on compassion fatigue and turnover intention. ${ }^{9,10}$ High work-burden, inefficient systemization, work-life conflict, and external control contribute to this phenomenon. In situations where medical needs rapidly emerge, such as the COVID-19 pandemic, this problem is exascerbated in both Korea and worldwide. ${ }^{11,12}$

Since many physicians, nurses, and other health professionals are at risk of burnout because of work-life imbalance, burnout in health professionals has been investigated in relation to treatment efficacy and adherence to medical ethics. ${ }^{13}$ Burned out medical professionals often express cynicism and pessimism toward treatment, which patients and their families could perceive as being cold, indifferent, and rude. ${ }^{14,15}$ Apathy regarding patients' needs and suffering may impair qualified health care activities. ${ }^{15}$ As empathetic communication is essential for high-quality humanistic care, ${ }^{16-21}$ affective, cognitive, and behavioral dimensions of empathetic communication require medical professionals to be competent enough to comprehend their patients' situations, feelings, and perspectives. ${ }^{22}$ Physician's empathy could enhance the patient's satisfaction and compliance, reduce anxiety and distress, and improve diagnostic accuracy and clinical outcomes, and eventually empower both patients and physicians. Consequently, doctors, nurses, and other healthcare providers need to be empathetic to provide optimal health care services. ${ }^{23}$

Presently, multi-faceted, and in-depth relations among WLB, burnout, and empathy of health professionals have not been fully investigated. We hypothesized that poor WLB would lead to greater burnout, which leads to lower empathy. The aims of this study were 1) to explore the quantitative relationships among WLB, burnout, and empathy, and 2) to investigate the role of the sub-dimensions of burnout in the relationship between WLB and empathy in health care professionals working at Seoul National University Hospital.

\section{METHODS}

\section{Study design and target population}

A cross-sectional, observational study was conducted from August to October 2018 using a convenience sampling method. The target population constituted healthcare professionals, including nurses and doctors in their residency training pro- grams, who had been working in the Seoul National University Hospital for more than six months. We recruited participants through an online, in-hospital recruiting board and offline postings that included a SurveyMonkey ${ }^{\mathrm{TM}}$ link on designated hospital boards. The participants were required to provide informed consent before participating, with a total of 213 health care professionals consenting, and responses being recorded anonymously. No financial compensation was given to participants. Among respondents, 108 provided incomplete responses, which resulted in an eligible sample of 105 . The study was approved by the Institutional Review Board of Seoul National University Hospital (IRB No C-1804-173-944).

\section{Work-life balance (WLB)}

Participants were asked to report their subjective WLB satisfaction on a scale from 0 to 100 with a single question, "How would you rate your satisfaction with the balance between work and life (out of 100)?” Despite there being several previous scales on WLB, "a (subjectively) perceived balance between work and the rest of life" as a definition per se for WLB was chosen as a single-item health indicator related to empathy and burnout since previous scales are not considered highly relevant to subjective well-being. ${ }^{24}$ Stress resilience and psychological attitude toward workload and private life are individually different, and subjective evaluation of WLB may be the most reliable measurement for subjective well-being. ${ }^{25}$ The "subjective" balance measure is not merely rating how satisfied or content providers are regarding time and workload. Respondents may engage in the process of reconsidering how they would ideally use their energy and time in a given situation. ${ }^{26}$

\section{Empathy scale}

The Jefferson Scale for Physician Empathy (JSPE) is widely used among health professionals and trainees. ${ }^{27,28}$ The 20 items ask about the respondent's current situation on a 7-level Likert scale from strongly disagree to strongly agree (total score range: $20-140$ ).

\section{Burnout scale}

Burnout was measured using the Korean version of the 22item Maslach Burnout Inventory (MBI), originally invented by Maslach and Jackson ${ }^{29}$ and formally validated in Korean. ${ }^{30}$ The inventory questions are answered based on the participant's current situation on a 7-point scale for each question. It is divided into three subscales, Emotional Exhaustion (nine items to measure feelings of being emotionally exhausted), Depersonalization (five items to measure an impersonal response to patients), and Personal Accomplishment (eight items to measure feelings of competence and achievement). 


\section{Mental health indices}

The brief Patient Health Questionnaire (PHQ-9), composed of nine items corresponding to the Diagnostic and Statistical Manual of Mental Disorders (DSM-IV)'s major depressive disorder criteria, was used to measure depressive symptoms over the past two weeks with a score ranging from 0 to 3 for each question. While the threshold score has varied in each study, a score of 8 or higher is considered to warrant clinical concern, and a higher score reflects even greater severity. ${ }^{31}$

The validated Korean version of the Beck Anxiety Inventory (BAI), a 21-item questionnaire listing anxiety symptoms in the past week, with ratings from 0 (not at all) to 3 (severe) for each item, was administered. The maximum score is 63 , and a score of 8 or higher was considered to represent mild anxiety. ${ }^{32,33}$ Measures of depressive symptoms and anxiety were included since they represent the leading causes of mental disability, costing the global economy US\$ 1 trillion each year in lost productivity. $^{34}$

Sleep problems over the past two weeks were assessed by the Korean version of the Insomnia Severity Index (ISI), composed of seven items on a 5-point Likert scale and validated in Korean.$^{35} \mathrm{~A}$ score of 15 or higher was considered to indicate insomnia. ${ }^{36}$

Possible current alcohol problems were also screened by the Korean version of the Alcohol Use Disorder Identification Test (AUDIT-K), with scores ranging from 0 to 40 . A score of 12 or higher signified an alcohol problem. ${ }^{37,38}$

\section{Other variables}

Demographic variables including age, sex, length of time working at the hospital, current department, education level, and marital status were gathered.

\section{Statistical analyses}

The mean values and standard deviations of subjective WLB, MBI, and JSPE were calculated. These values were calculated according to gender, age $(<30,30-39,40-49$, and $\geq 50$ years), work duration at the hospital (0.5-2, 3-4, 5-9, $\geq 10$ years), education level (up to college and above college), and marital status (single and married). We used independent t-tests and analysis of variance (ANOVA) to compare the differences in scores of WLB, MBI, and JSPE according to the demographic variables.

To test the differences in mental health, including anxiety (BAI $\geq 16$ ), depressive symptoms (PHQ- $9 \geq 8$ ), insomnia (ISI $\geq 10$ ), and alcohol use problems (AUDIT-K $\geq 12$ ) according to WLB, participants were classified into good WLB group (scored $\geq 50$ th percentile) and bad WLB group (score $<50$ th percentile) to increase the contrast between the two groups. A chi-square test was used to examine the differences be- tween mental health indices, according to the WLB group. Next, multivariate linear regression models were constructed to investigate the association between WLB and empathy. We used quartiles of subjective WLB score class (categorical variable) as the independent variable and JSPE score (20140, continuous) as the dependent variable. In Model 1, we adjusted for demographic variables, including work duration, education, and marital status. In Model 2, we additionally adjusted for mental health indices, such as anxiety, depressive symptoms, insomnia, and alcohol use problems. Finally, in Model 3, three dimensions of MBI (Burnout, Depersonalization, and Personal Achievement) were further adjusted.

Finally, a mediation model was hypothesized and explored for the role of the sub-dimensions of MBI between WLB and empathy. Bootstrapped mediation analyses were performed to examine the direct and indirect effects of WLB on empathy through the MBI subdomains..$^{39}$ Mediation analyses involve the estimation of three equations: ${ }^{40}$

$$
\begin{aligned}
& \text { Equation 1: } Y=b_{11}+X^{\prime} c_{12} \\
& \text { Equation 2: } M=b_{21}+X^{\prime} c_{22} \\
& \text { Equation 3: } Y=b_{31}+X^{\prime} c_{32}+M^{\prime} c_{33}
\end{aligned}
$$

In this analysis, $b_{11}, b_{21}$ and $b_{31}$ stand for constants, while $c_{12}$, $c_{22}, c_{32}$ and $c_{33}$ refer to the mean coefficients. The coefficient in Equation $1\left(c_{12}\right)$ was considered as the direct effect of the causal variable $\mathrm{X}^{\prime}$ (WLB score) on the outcome variable Y (JSPE score), without the effect of the mediator M (subdomains of Maslach Burnout Inventory). It presents 95\% confidence intervals (two-tailed $\mathrm{p}<0.05$ ) of indirect effects in the model. Mediation analyses were performed with adjustments for age, work duration, education, marital status, and mental health indices (i.e., anxiety, depressive symptoms, insomnia, and alcohol use problems). Statistical analyses were performed using SAS (Version 9.4; SAS Institute Inc., Cary, NC, USA).

\section{RESULTS}

\section{Demographic and clinical characteristics}

Table 1 shows the characteristics of the study participants. Although most incomplete respondents discontinued the survey at an early stage, no difference in demographic characteristics was found between complete and incomplete respondents. A total of 105 health professionals participated in our study, and 96 (91.4\%) were female. Most participants were below 30 (51.4\%), had worked for two years or less (50.5\%), were college-educated or below (78.1\%), and single (71.4\%). The mean subjective WLB score was $50.9(\mathrm{SD}=24.3)$, and the mean JSPE score was $88.3(\mathrm{SD}=16.4)$. The subjective WLB 
Table 1. The work-life balance, empathy and burnout scores by the characteristics of study participants

\begin{tabular}{|c|c|c|c|c|c|c|c|c|c|c|c|}
\hline & \multirow{2}{*}{$\mathrm{N}(\%)$} & \multirow{2}{*}{ df } & \multicolumn{3}{|c|}{ Subjective WLB score } & \multicolumn{3}{|c|}{ MBI score } & \multicolumn{3}{|c|}{ JSPE score } \\
\hline & & & Mean \pm SD & tor $\mathrm{F}$ & $\mathrm{p}^{*}$ & Mean \pm SD & t or $F$ & $\mathrm{p}^{*}$ & Mean \pm SD & t or $F$ & $\mathrm{p}^{*}$ \\
\hline Total & $105(100)$ & & $50.9 \pm 24.3$ & & & $63.5 \pm 25.5$ & & & $88.3 \pm 16.4$ & & \\
\hline \multicolumn{12}{|l|}{ Sex } \\
\hline Male & $9(8.6)$ & 103 & $55.6 \pm 27.6$ & 0.60 & 0.547 & $51.1 \pm 19.5$ & -1.53 & 0.129 & $92.6 \pm 16.2$ & 0.80 & 0.424 \\
\hline Female & $96(91.4)$ & & $50.4 \pm 24.1$ & & & $64.6 \pm 25.8$ & & & $87.9 \pm 16.5$ & & \\
\hline \multicolumn{12}{|l|}{ Age (y) } \\
\hline$<30$ & $54(51.4)$ & $(3,101)$ & $41.7 \pm 22.5$ & 6.94 & $<0.001$ & $73.3 \pm 25.0$ & 7.13 & $<0.001$ & $81.9 \pm 15.3$ & 6.74 & $<0.001$ \\
\hline $30-39$ & $36(34.3)$ & & $58.2 \pm 23.0$ & & & $55.3 \pm 21.9$ & & & $96.0 \pm 16.1$ & & \\
\hline $40-49$ & $11(10.5)$ & & $63.5 \pm 22.6$ & & & $51.4 \pm 21.2$ & & & $94.0 \pm 12.0$ & & \\
\hline$\geq 50$ & $4(3.8)$ & & $74.5 \pm 12.2$ & & & $37.8 \pm 22.0$ & & & $90.8 \pm 13.2$ & & \\
\hline \multicolumn{12}{|l|}{ Work duration (y) } \\
\hline $0.5-2$ & $53(50.5)$ & $(3,101)$ & $35.9 \pm 19.3$ & 10.78 & $<0.001$ & $79.5 \pm 23.2$ & 10.82 & $<0.001$ & $80.9 \pm 14.3$ & 4.91 & 0.003 \\
\hline $3-4$ & $25(23.8)$ & & $52.5 \pm 25.2$ & & & $60.8 \pm 24.9$ & & & $88.0 \pm 17.0$ & & \\
\hline $5-9$ & $18(17.1)$ & & $56.0 \pm 23.2$ & & & $58.3 \pm 21.2$ & & & $93.1 \pm 17.3$ & & \\
\hline$\geq 10$ & $9(8.6)$ & & $68.6 \pm 17.6$ & & & $45.3 \pm 19.8$ & & & $95.6 \pm 13.5$ & & \\
\hline \multicolumn{12}{|l|}{ Education } \\
\hline$\leq$ College & $82(78.1)$ & 103 & $48.3 \pm 24.2$ & -2.10 & 0.038 & $66.9 \pm 25.6$ & 2.70 & 0.008 & $85.9 \pm 16.0$ & -3.03 & 0.003 \\
\hline Above college & $23(21.9)$ & & $60.1 \pm 22.9$ & & & $51.1 \pm 21.5$ & & & $97.2 \pm 15.4$ & & \\
\hline \multicolumn{12}{|l|}{ Marital status } \\
\hline Single & $75(71.4)$ & 103 & $46.4 \pm 22.8$ & -3.10 & 0.003 & $68.4 \pm 24.4$ & 3.34 & 0.001 & $86.4 \pm 16.8$ & 1.31 & 0.055 \\
\hline Married & $30(28.6)$ & & $62.0 \pm 24.6$ & & & $50.9 \pm 24.1$ & & & $93.2 \pm 14.7$ & & \\
\hline
\end{tabular}

*to test the differences among variable groups, Student's t-test (2 groups) or ANOVA ( $\geq 3$ groups) was performed. WLB: work-life balance, JSPE: Jefferson’s Scale of Physician Empathy, MBI: Maslach Burnout Inventory

score was positively and significantly associated with older age, longer work duration, higher education, and married status. Higher MBI scores were related to a younger age, shorter work duration, lower education, and unmarried status. Higher JSPE scores were also significantly associated with older age, longer work duration, and higher education level, but marital status was not significantly associated.

Table 2 shows mental health indices by subjective WLB score groups. Compared to the low WLB group, the high WLB group consisted of fewer nurses with anxiety ( $44.8 \%$ vs. $21.3 \%$, $\mathrm{p}<0.011$ ), depressive symptoms ( $65.5 \%$ vs. $29.8 \%, \mathrm{p}<0.001$ ), and insomnia ( $70.7 \%$ vs. $51.1 \%, \mathrm{p}=0.023$ ). WLB level groups did not significantly differ in terms of alcohol use problems.

\section{Multivariate regression analyses: the association between WLB and empathy}

Table 3 presents the association between WLB and empathy, which were analyzed using multivariate linear regression models. Model 1 used age, work duration, education level, and marital status as covariates. The results of regression indicated that subjective WLB group and the covariates explained 29.0\% of the variance $\left[R^{2}=0.29, F(8,104)=4.98, p<0.001\right]$. In this model, compared to the best subjective WLB group (fourth quartile), the good group (third quartile), poor group (second quartile), and the worst group (first quartile) had significantly
Table 2. Mental health indices according to the subjective worklife balance

\begin{tabular}{|c|c|c|c|}
\hline & $\begin{array}{c}\text { Good WLB } \\
(\geq 50 \text { th percentile }) \\
(\mathrm{N}=47)\end{array}$ & $\begin{array}{c}\text { Bad WLB } \\
(<50 \text { th percentile }) \\
(\mathrm{N}=58)\end{array}$ & \multirow[t]{2}{*}{$\begin{array}{c}\text { p- } \\
\text { value* }^{*}\end{array}$} \\
\hline & $\mathrm{N}(\%)^{\dagger}$ & $\mathrm{N}(\%)^{\dagger}$ & \\
\hline Anxiety & & & 0.011 \\
\hline $\mathrm{BAI} \geq 16$ & $10(21.3)$ & $26(44.8)$ & \\
\hline $\mathrm{BAI}<16$ & $37(78.7)$ & $32(55.2)$ & \\
\hline \multicolumn{2}{|c|}{ Depressive symptoms } & & $<0.001$ \\
\hline PHQ- $9 \geq 8$ & $14(29.8)$ & $38(65.5)$ & \\
\hline PHQ-9 $<8$ & $33(70.2)$ & $20(34.5)$ & \\
\hline Insomnia & & & 0.023 \\
\hline ISI $\geq 10$ & $24(51.1)$ & $41(70.7)$ & \\
\hline ISI $<10$ & $23(48.9)$ & $17(29.3)$ & \\
\hline \multicolumn{2}{|c|}{ Alcohol use disorder } & & 0.095 \\
\hline AUDIT-K $\geq 12$ & $6(12.8)$ & $15(25.9)$ & \\
\hline AUDIT-K <12 & $41(87.2)$ & $43(74.1)$ & \\
\hline
\end{tabular}

*chi-square tests were performed, ${ }^{\dagger}$ percent in column. WLB: worklife balance, PHQ-9: Patient Health Questionnaire-9, ISI: Insomnia Severity Index, BAI: Beck's Anxiety Inventory, AUDIT-K: Korean version of the Alcohol Use Disorder Identification Test

lower JSPE scores at $-13.32(\mathrm{p}=0.005),-12.35(\mathrm{p}=0.005)$, and $-21.50(\mathrm{p}<0.001)$, respectively. Higher education level was significantly associated with a higher empathy level. Model 2 
HY Seo et al.

Table 3. Results of multivariate linear regression models for the association between health professionals' work-life balance and empathy

\begin{tabular}{|c|c|c|c|c|c|c|c|c|c|}
\hline \multirow{2}{*}{ Variables } & \multicolumn{3}{|c|}{ Model 1} & \multicolumn{3}{|c|}{ Model 2} & \multicolumn{3}{|c|}{ Model 3} \\
\hline & $\beta$ & SE & p-value & $\beta$ & SE & $\mathrm{p}$-value & $\beta$ & SE & $\mathrm{p}$-value \\
\hline \multicolumn{10}{|l|}{ Subjective work-life balance } \\
\hline \multicolumn{10}{|l|}{ Best (4Q, Reference) } \\
\hline Good (3Q) & -13.32 & 4.63 & 0.005 & -11.21 & 4.62 & 0.017 & -6.04 & 5.51 & 0.276 \\
\hline Poor (2Q) & -12.35 & 4.25 & 0.005 & -9.06 & 4.74 & 0.059 & -3.83 & 4.71 & 0.417 \\
\hline Worst (1Q) & -21.50 & 4.35 & $<0.001$ & -16.67 & 5.21 & 0.002 & -5.95 & 4.43 & 0.182 \\
\hline Age & 0.19 & 0.34 & 0.561 & 0.06 & 0.38 & 0.878 & 0.13 & 0.35 & 0.716 \\
\hline \multicolumn{10}{|l|}{ Work duration (vs. 0-2 years) } \\
\hline $3-4$ years & -0.15 & 3.89 & 0.969 & 1.00 & 3.78 & 0.793 & -3.72 & 3.55 & 0.298 \\
\hline 5-9 years & 5.83 & 4.20 & 0.168 & 6.16 & 4.33 & 0.157 & 1.81 & 4.37 & 0.680 \\
\hline$\geq 10$ years & 1.02 & 7.37 & 0.890 & 0.24 & 7.66 & 0.975 & -3.21 & 6.66 & 0.631 \\
\hline Education (above college vs. up to college) & 8.10 & 3.86 & 0.038 & 8.18 & 3.72 & 0.030 & 5.47 & 3.22 & 0.092 \\
\hline Married (vs. single) & -2.54 & 4.07 & 0.534 & -0.87 & 3.72 & 0.164 & -3.29 & 3.92 & 0.404 \\
\hline Anxiety (BAI $\geq 16)$ & & & & -5.22 & 3.72 & 0.164 & -2.21 & 3.17 & 0.487 \\
\hline Depressive symptoms (PHQ-9 $\geq 8$ ) & & & & -5.8 & 4.01 & 0.152 & -0.86 & 3.49 & 0.807 \\
\hline Insomnia (ISI $\geq 10$ ) & & & & 3.02 & 3.31 & 0.363 & 2.97 & 2.72 & 0.276 \\
\hline Alcohol use disorder (AUDIT-K $\geq 12$ ) & & & & -4.11 & 3.64 & 0.261 & -1.28 & 3.42 & 0.708 \\
\hline Emotional exhaustion & & & & & & & -0.19 & 0.21 & 0.364 \\
\hline Depersonalization & & & & & & & -0.49 & 0.25 & 0.056 \\
\hline Personal achievement & & & & & & & 0.58 & 0.16 & $<0.001$ \\
\hline Adjusted $\mathrm{R}^{2}$ & 0.29 & & & 0.35 & & & 0.54 & & \\
\hline Degree of freedom & $(8,104)$ & & & $(12,104)$ & & & $(15,104)$ & & \\
\hline F value & 4.98 & & & 5.89 & & & 8.42 & & \\
\hline
\end{tabular}

PHQ-9: Patient Health Questionnaire-9, ISI: Insomnia Severity Index, BAI: Beck’s Anxiety Inventory, AUDIT-K: the Korean version of Alcohol Use Disorder Identification Test

used anxiety, depressive symptoms, insomnia, and alcohol use problem as additional covariates to Model 1 . The results of regression indicated that Model 2 explained $34.6 \%$ of the variance, $\mathrm{R}^{2}=0.35, \mathrm{~F}(12,104)=5.89, \mathrm{p}<0.001$. In Model 2, the good group $(\beta=-11.21, p=0.017)$ and the worst group $(\beta=-$ $16.67, \mathrm{p}=0.002$ ) also had significantly lower JSPE scores than the best group. In Model 3, three dimensions of the burnout scale (Emotional Exhaustion, Depersonalization, and Personal Achievement) were additionally used as covariates to Model 2, and Model 3 explained $54.0 \%$ of the variance, $\mathrm{R}^{2}=0.54$, $\mathrm{F}(15,104)=8.42, \mathrm{p}<0.001$. In this model, the association between subjective WLB and JSPE score disappeared. Only personal achievement among the burnout sub-dimensions was associated with a better JSPE score $(\beta=0.56, \mathrm{p}<0.001)$.

\section{Mediation analyses: WLB, empathy, and burnout}

Figure 1 shows the results of mediation analyses performed to investigate the indirect effects of subjective WLB on empathy through the three sub-dimensions of burnout. We found significant mediating effects of burnout between WLB and Empathy. The direct effect of WLB on Empathy was not significant ( $\beta=0.06,95 \% \mathrm{CI}:-0.06-0.20, \mathrm{p}=0.450$ ); however, the total indirect effect was significant $(\beta=0.17,95 \%$
CI: 0.06-0.29, $\mathrm{p}=0.003)$. More specifically, Personal Achievement $(\mathrm{p}=0.011)$ and Depersonalization $(\mathrm{p}=0.047)$ among the subscales of burnout were found to be mediating variables.

\section{DISCUSSION}

To our knowledge, this is the first study investigating WLB in relation to empathy and burnout status of health care workers. In our cross-sectional study, we found that poor WLB was significantly related to a low degree of empathy, and this association was mediated by personal achievement, a sub-dimension of burnout. Those with the highest score on WLB also reported the best score in the Jefferson scale of empathy, and burnout was the parameter that mediated between these two factors.

\section{Association between burnout versus WLB \& between burnout versus empathy}

The relationship between subjective WLB and burnout in our study is compatible with other studies. A study targeting engineers and nurses reported that burnout is a direct consequence of work-home conflict for both groups. ${ }^{41}$ Another study with 750 Italian nurses found that work-family conflict is significantly linked to burnout. ${ }^{42}$ Although most studies 


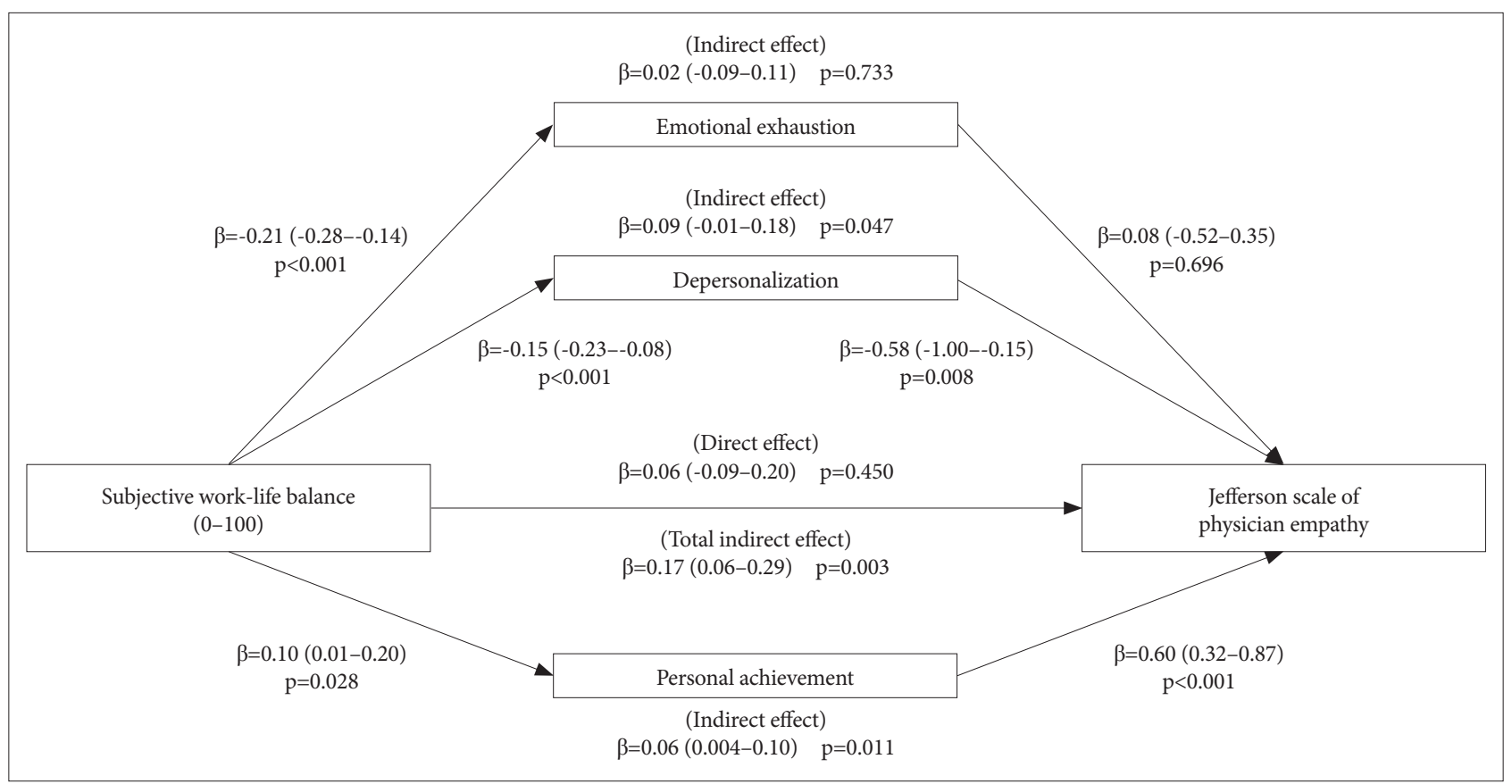

Figure 1. Results of mediation analyses.

were cross-sectional, which cannot test for causality, a longitudinal study with 242 Spanish soldiers has provided some insight on the issue, showing the reciprocal relationship between work-family conflict and emotional exhaustion as a facet of burnout. ${ }^{43}$ Although the causal linkage between subjective WLB and qualitative/quantitative measurements were not confirmed in many comprehensive studies, life balance measures individuals' overall life pattern and represents one's life system holistically. ${ }^{26}$ Instead of controversial measures of WLB, we chose the simplest form of subjective evaluation on WLB. Neurotic people may complain about disturbed WLB despite having objectively healthy lifestyles. Conversely, optimists may handle heavy schedules and workloads with serene perspectives if they believe their lives are fulfilling and meaningful. ${ }^{44}$ Regarding the limitless confounding factors in judging WLB, a subjective assessment may be the most reliable variable for predicting burnout.

The negative association between burnout and empathy among primary physicians and nurses was established in other studies. A recent systematic review revealed that burnout mostly has a negative association with empathy, with 8 out of 10 studies included showing a negative correlation. ${ }^{45}$

\section{Feelings of personal achievement \& higher educational attainment: a possible booster of empathy?}

Among burnout scales, Personal Achievement items were found to be significantly related to empathy, and higher education level was significantly associated with higher empathy levels. Several studies showed similar results where high levels of empathy were reported to correlate with the personal accomplishment factors in the MBI. ${ }^{46-48}$ The personal accomplishment factor is related to intrinsic personal factors, such as being positive, happy, and sociable. A feeling of strong personal accomplishment may lower the risk of compassion fatigue and burnout, which are often labeled as emotional labor. ${ }^{49}$

In our study, those with higher education and older people who have a better chance of personal accomplishment reported being more empathetic and having less burnout, which is compatible with the above-mentioned findings. To have better hospital management, various strategies to enhance empowerment, such as active participation in decision-making, research on important issues, promotion, and prizes, should be developed.

\section{Need for educational approach \& working environment changes for healthcare professionals}

Our finding showed a medium level of burnout compared to other studies targeting health professionals. ${ }^{50,51}$ A few studies reported tentative findings on interventions to increase empathy and prevent burnout. Leisure and exercise programs to improve quality of life were developed to prevent burnout among physicians. ${ }^{52,53}$ Preventative intervention focuses on developing workers' engagement and putting more emphasis on organizations to take some responsibility in the detection and prevention of burnout. ${ }^{54}$ West and colleagues noted that individual (e.g., mindfulness, discussion, and stress management) and organizational (e.g., work environment) interventions similarly produced substantial improvements in burnout. ${ }^{55} \mathrm{~A}$ recent me- 
ta-analysis confirmed that burnout in health care professionals is a problem of the whole organization rather than of individuals. ${ }^{56}$ The Mayo clinic presented nine organizational strategies to reduce physicians' burnout by emphasizing more flexibility and facilitating leadership to ensure members' resiliency and self-care. ${ }^{57}$ The suggested strategies include 1)Acknowledging and assessing the problem, 2) Harnessing the power of leadership, 3) Developing and implementing targeted interventions, 4) Cultivating the community at work, 5) Using rewards and incentives wisely, 6) Aligning values and strengthening the work culture, 7) Promoting flexibility and work-life integration, 8) Providing resources to promote resilience and self-care, and 9) Facilitating and funding organizational science.

Fundamental reform of hospital settings has been suggested, decreasing the burden of documentation and delivery system. Physicians need to be trained as coaching/mentoringleaders who keep their team informed and enable them to actively participate in improving their work environment. ${ }^{58}$ The National Academy of Medicine (NAM) $)^{59}$ launched a national Action Collaborative on Clinician Well-Being and Resilience. NAM offers various workgroups for sharing information, optimal team-based care and documentation, and tool kits for a productive relationship. ${ }^{60}$

Compassion fatigue or professionals who become mentally ill because of excessive workload often cause inefficient and inhumane health care, which brings about a less safe hospital environment because of anger issues. Dual responsibilities, both at home and work, may lead to burnout symptoms, which may explain the significant global shortage of health care workers. Nurses who are relatively less empathetic, not being aware of their burnout or mental illness may provoke various conflicts and eventually become involved with medical accidents, poor service, malpractice suits, and violent incidents. ${ }^{61}$ Over the next 20 years, many health professionals may quit their jobs if proper preventive measures against burnout and emotional or physical overwork are not undertaken, which could threaten the wellness of society despite advancements in technological development. ${ }^{62}$

\section{Limitations}

This study has several limitations. Although it was hypothesized that WLB would affect burnout, and burnout would affect empathy, the possibility of reverse causality should be considered. Burned-out workers may not try to pursue WLB. Workers who have less empathy for other psychological reasons or personality structures may feel more burnout related to their sacrificial job requirements. Sound evidence is needed in prospective cohort studies concerning WLB, burnout, and empathy. Second, although subjective satisfaction in WLB is critical when considering both intrinsic resilience and the external en- vironments, quantification of WLB with a well-validated and objective scale is necessary for clarifying the relationship. Additionally, the item assessing WLB did not specify the specific period of time being measured, which may result in variability between respondents. Third, an integrated analysis of the determinants of empathy could not be performed in our study. As one of our hypotheses was to test the mediating roles of burnout in the relationship between WLB and empathy, we did not assess several empathy-related variables, such as personal traits or emotional intelligence. However, we performed mediation analyses adjusting for demographic variables and mental health indices. In the future, investigating the integrated analysis for empathy using structural equation modeling while considering various potential determinants of empathy is needed. Finally, most of the respondents were female; hence, gender differences could not be explored. Women caring for children and managing the household in a traditional society may be either more vulnerable or more resilient, which was not confirmed by this study. Fourth, the targeted workers, working in a big general hospital in Seoul, where the workload is typically very high and competitive, may have different profiles from workers in other environments.

\section{Conclusions}

We found that WLB correlated positively with empathy and personal achievement, with one sub-dimensions of burnout appearing to be a significant mediating factor. WLB was negatively associated with burnout, but the causal nature of the relationship between the two factors was not confirmed. Theoretically, subjective feelings of work-life imbalance may cause burnout symptoms, and burnout symptoms may bring about an imbalanced work-life balance in health care professionals. Further longitudinal studies are needed to explore the causal relationship between these variables.

\section{Acknowledgments}

This study was supported by donated grants to Seoul national university hospital under the name of "the Rhie blended family Foudation" with support from Dr. Rhie SooBum. Dr. Rhie JungYeon and scretary-general Rhie JuneKyue (Grant No: 900-00086).

\section{Conflicts of Interest}

The authors have no potential conflicts of interest to disclose.

\section{Author Contributions}

Conceptualization: Nami Lee. Data curation: Hwo-Yeon Seo. Formal analysis: Dong-Wook Lee. Funding acquisition: Nami Lee. Investigation: Hwo-Yeon Seo. Methodology: Yun-Chul Hong. Project administration: Nami Lee. Resources: Sung-jun Cho. Software: Dong-Wook Lee. Supervision: Nami Lee. Validation: Je-Yeon Yoon. Visualization: Dong-Wook Lee. Writing_original draft: Hwo-Yeon Seo. Writing_review \& editing: Soohyun Nam. 


\section{ORCID iDs}

Hwo-Yeon Seo

Dong-Wook Lee

Soohyun Nam

Sung-jun Cho

Je-Yeon Yun

Yun-Chul Hong

Nami Lee https://orcid.org/0000-0002-0523-5816

https://orcid.org/0000-0002-4023-3269

https://orcid.org/0000-0002-1342-2769

https://orcid.org/0000-0001-6981-0931

https://orcid.org/0000-0002-5531-2410

https://orcid.org/0000-0001-9010-7271

https://orcid.org/0000-0001-6971-7542

\section{REFERENCES}

1. Lee KM. 52-hour work week takes effect. Seoul, Korea Times. Available at: http://www.koreatimes.co.kr/www/nation/2019/10/371_251549. html. Accessed October 14, 2019.

2. Yang JW, Suh C, Lee CK, Son BC. The work-life balance and psychosocial well-being of South Korean workers. Ann Occup Environ Med 2018;30:38.

3. Park JB, Nakata A, Swanson NG, Chun H. Organizational factors associated with work-related sleep problems in a nationally representative sample of Korean workers. Int Arch Occup Environ Health 2013;86:211222.

4. Virtanen M, Jokela M, Nyberg ST, Madsen IE, Lallukka T, Ahola K, et al. Long working hours and alcohol use: systematic review and metaanalysis of published studies and unpublished individual participant data. BMJ 2015;350:g7772.

5. Park JH, Yoon SJ, Lee HY, Cho HS, Lee JY, Eun SJ, et al. Estimating the burden of psychiatric disorder in Korea. J Prev Med Public Health 2006;39:39-45

6. Cho T. A sobering look at South Korea's drinking culture. Available at: http://www.themonsoonproject.org/a-sobering-look-at-south-koreasdrinking-culture/. Accessed May 15, 2019.

7. Oh SJ, Jung SM. Development of the role of teaching hospitalists in the education of residents. J Korean Med Assoc 2019;62:573-576.

8. Song SY. $80 \%$ of Doctors Experience Burnout: A survey. Seoul, Korea Medical Review. Available at: http://www.koreabiomed.com/news/articleView.html?idxno=4952/. Accessed October 14, 2019.

9. June KJ, Byun SW. Nurses' burnout research throughout the past 10 years in Korea. J Korean Acad Nurs Admi 2009;15:305-313.

10. Kim K, Han Y, Kwak Y, Kim JS. Professional quality of life and clinical competencies among Korean nurses. Asian Nurs Res 2015;9:200-206.

11. Roy L. Doctor, heal thyself: physician burnout in the wake of Covid-19. Forbes 2020 May 17. Available at: https://www.forbes.com/sites/ lipiroy/2020/05/17/doctor-heal-thyself-physician-burnout-in-thewake-of-covid-19/\#2ea7c489213c. Accessed June 27, 2020.

12. Arin K. Korea's virus fight 'may drag on for months, years.' The Korea Herald 2020 Apr 6. Available at: http://www.koreaherald.com/view. php?ud=20200405000242\&ACE_SEARCH=1. Accessed June 27,2020.

13. Yuguero O, Ramon Marsal J, Esquerda M, Vivanco L, Soler-González J. Association between low empathy and high burnout among primary care physicians and nurses in Lleida, Spain. Eur J Gen Pract 2017;23:410.

14. Özler DE, Atalay CG. A research to determine the relationship between organizational cynicism and burnout levels of employees in health sector. Bus Manag Rev 2011;1:26-38.

15. Maslach C, Schaufeli WB, Leiter MP. Job burnout. Annu Rev Psychol 2001;52:397-422.

16. Cole-King A, Gilbert P. Compassionate care: the theory and reality. J Holist Healthc 2011;8:32.

17. Buszewicz M, Pistrang N, Barker C, Cape J, Martin J. Patients' experiences of GP consultations for psychological problems: a qualitative study. Br J Gen Pract 2006;56:496-503.

18. Hojat M, Louis DZ, Markham FW, Wender R, Rabinowitz C, Gonnella JS. Physicians' empathy and clinical outcomes for diabetic patients. Acad Med 2011;86:359-364.

19. Hojat M, Louis DZ, Maxwell K, Markham FW, Wender RC, Gonnella
JS. A brief instrument to measure patients' overall satisfaction with primary care physicians. Fam Med 2011;43:412-417.

20. Levinson W, Roter D. Physicians' psychosocial beliefs correlate with their patient communication skills. J Gen Intern Med 1995;10:375379.

21. van Dulmen S, van den Brink-Muinen A. Patients' preferences and experiences in handling emotions: a study on communication sequences in primary care medical visits. Patient Educ Couns 2004;55:149-152.

22. Derksen F, Bensing J, Lagro-Janssen A. Effectiveness of empathy in general practice: a systematic review. Br J Gen Pract 2013;63:e76-e84.

23. Aiken LH, Clarke SP, Sloane DM, Sochalski J, Silber JH. Hospital nurse staffing and patient mortality, nurse burnout, and job dissatisfaction. JAMA 2002;288:1987-1993.

24. Pichler F. Determinants of work-life balance: shortcomings in the contemporary measurement of WLB in large-scale surveys. Soc Indic Res 2009;92:449-469.

25. Guest DE. Perspectives on the study of work-life balance. Soc Sci Inform 2002;41:255-279.

26. Sheldon KM, Cummins R, Kamble S. Life balance and well-being: testing a novel conceptual and measurement approach. J Pers 2010;78: 1093-1134.

27. Hojat M, Mangione S, Nasca TJ, Cohen MJM, Gonnella JS, Erdmann JB, et al. The Jefferson scale of physician empathy: development and preliminary psychometric data. Educ Psychol Meas 2001;61:349-365.

28. Kang WS, Kim YH, Jang HI. An investigation on reliability and validity of the Korean Physician Empathy Rating Scale. Korean J Psychopathol 2006;15:11-18.

29. Maslach C, Jackson SE. The measurement of experienced burnout. J Organ Behav 1981;2:99-113.

30. Kang JH, Kim CW. Evaluating applicability of Maslach Burnout Inventory among university hospitals nurses. Korean J Adult Nurs 2012;24:3137.

31. Kroenke K, Spitzer RL, Williams JBW. The PHQ-9: validity of a brief depression severity measure. J Gen Intern Med 2001;16:606-613.

32. Beck AT, Steer RA. Manual for the Beck Anxiety Inventory. San Antonio, TX: Psychological Corporation; 1990.

33. Yook S, Kim Z. A clinical study on the Korean version of beck anxiety inventory: comparative study of patient and non-patient. Korean J Clin Psychol 1997;16:185-197.

34. World Health Organization. Mental health in the workplace. Information sheet. Available at: https://www.who.int/mental_health/in_the_ workplace/en/. Accessed May 15, 2019.

35. Cho YW, Song ML, Morin CM. Validation of a Korean version of the Insomnia Severity Index. J Clin Neurol 2014;10:210-215.

36. Bastien CH, Vallières A, Morin CM. Validation of the Insomnia Severity Index as an outcome measure for insomnia research. Sleep Med 2001;2:297-307.

37. Lee BO, Lee CH, Lee PG, Choi NJ, Namkoong K, Lee B, et al. Development of Korean version of alcohol use disorders identification test (AUDIT-K): its reliability and validity. J Korean Acad Addict Psychiatry 2000;4:83-92.

38. Saunders JB, Aasland OG, Babor TF, de la Fuente JR, Grant M. Development of the Alcohol Use Disorders Identification Test (AUDIT): WHO Collaborative Project on Early Detection of Persons with Harmful Alcohol Consumption-II. Addiction 1993;88:791-804.

39. Hayes AF. PROCESS: A Versatile Computational Tool for Observed Variable Mediation, Moderation, and Conditional Process Modeling. KS: University of Kansas; 2012.

40. Baron RM, Kenny DA. The moderator-mediator variable distinction in social psychological research: conceptual, strategic, and statistical considerations. J Pers Soc Psychol 1986;51:1173-1182.

41. Bacharach SB, Bamberger P, Conley S. Work-home conflict among nurses and engineers: mediating the impact of role stress on burnout and satisfaction at work. J Organ Behav 1991;12:39-53.

42. Camerino D, Sandri M, Sartori S, Conway PM, Campanini P, Costa G. 
Shiftwork, work-family conflict among Italian nurses, and prevention efficacy. Chronobiol Int 2010;27:1105-1123.

43. Rubio C, Osca A, Recio P, Urien B, Peiró JM. Work-family conflict, self-efficacy, and emotional exhaustion: a test of longitudinal effects. Rev Psicol Trab Organ 2015;31:147-154.

44. Khan OF, Fazili AI. Work-life balance: a conceptual review. J Strateg Hum Resour Manag 2016;5:20-25.

45. Wilkinson H, Whittington R, Perry L, Eames C. Examining the relationship between burnout and empathy in healthcare professionals: a systematic review. Burn Res 2017;6:18-29.

46. Ferri P, Guerra E, Marcheselli L, Cunico L, Di Lorenzo R. Empathy and burnout: an analytic cross-sectional study among nurses and nursing students. Acta Biol Med 2015;86(Suppl 2):104-115.

47. Lamothe M, Boujut E, Zenasni F, Sultan S. To be or not to be empathic: the combined role of empathic concern and perspective-taking in understanding burnout in general practice. BMC Fam Pract 2014;15:1-7.

48. Omdahl BL, O’Donnell C. Emotional contagion, empathic concern and communicative responsiveness as variables affecting nurses' stress and occupational commitment. J Adv Nurs 1999;29:1351-1359.

49. Zeidner M, Hadar D, Matthews G, Roberts RD. Personal factors related to compassion fatigue in health professionals. Anxiety Stress Coping 2013;26:595-609.

50. Goodman MJ, Schorling JB. A mindfulness course decreases burnout and improves well-being among healthcare providers. Int J Psychiatry Med 2012;43:119-128.

51. Volpe U, Luciano M, Palumbo C, Sampogna G, Del Vecchio V, Fiorillo A. Risk of burnout among early career mental health professionals. J Psychiatr Ment Health Nurs 2014;21:774-781.

52. Dieser RB, Edginton CR, Ziemer R. Decreasing patient stress and physician/medical workforce burnout through health care environments: uncovering the serious leisure perspective at Mayo Clinic's campus in
Rochester, Minnesota. Mayo Clin Proc 2017;92:1080-1087.

53. Weight CJ, Sellon JL, Lessard-Anderson CR, Shanafelt TD, Olsen KD, Laskowski ER. Physical activity, quality of life, and burnout among physician trainees: the effect of a team-based, incentivized exercise program. Mayo Clin Proc 2013;88:1435-1442.

54. Maslach C. Burnout and engagement in the workplace: new perspectives. Eur Health Psychol 2011;13:44-47.

55. West CP, Dyrbye LN, Erwin PJ, Shanafelt TD. Interventions to prevent and reduce physician burnout: a systematic review and meta-analysis. Lancet 2016;388:2272-2281.

56. Panagioti M, Panagopoulou E, Bower P, Lewith G, Kontopantelis E, Chew-Graham C, et al. Controlled interventions to reduce burnout in physicians: a systematic review and meta-analysis. JAMA Intern Med 2017;177:195-205

57. Shanafelt TD, Noseworthy JH. Executive leadership and physician well-being: nine organizational strategies to promote engagement and reduce burnout. Mayo Clin Proc 2017;92:129-146.

58. Shanafelt TD, Dyrbye LN, West CP. Addressing physician burnout: the way forward. JAMA 2017;317:901-902.

59. National Academy of Medicine (NAM). Action collaborative on clinician well-being and resilience. Available at: https://nam.edu/actioncollaborative-on-clinician-well-being-and-resilience-network-organizations/. Accessed October 14, 2019.

60. Dzau VJ, Kirch DG, Nasca TJ. To care is human-Collectively confronting the clinician-burnout crisis. N Engl J Med 2018;378:312-314.

61. Klimecki OM, Vuilleumier P, Sander D. The impact of emotions and empathy-related traits on punishment behavior: Introduction and validation of the inequality game. PLoS One 2016;11:e0151028.

62. The Lancet. No health workforce, no global health security. Lancet 2016;387:2063. 